\title{
Clinical characteristics and predictors of mortality in patients with normal wall thickness cardiac amyloidosis
}

\author{
Sabha Bhatti, Evan Watts, Srikanth Vallurupalli, Tarun Pandey, Abdul Hakeem \\ From 19th Annual SCMR Scientific Sessions \\ Los Angeles, CA, USA. 27-30 January 2016
}

\section{Background}

Clinical features and prognosis of Cardiac amyloidosis (CA) with normal wall thickness (NWT) have not been well elucidated.

\section{Methods}

305 patients with multiple myeloma undergoing cardiac MRI for suspected amyloidosis were consecutively enrolled. 251 patients underwent gadolinium study. Increased wall thickness was defined as left ventricular wall thickness $>12 \mathrm{~mm}$ on CMR.

\section{Results}

74 patients (29\%) had gadolinium enhancement pattern consistent with CA on CMR (31\% with NWT). With endomyocardial biopsy as the gold standard, amyloid pattern on CMR (LGE+) had sensitivity and negative predictive values of $100 \%$; specificity and positive predictive values of $80 \%$ and $81 \%$ with an AUC 0.9 for CA. There was no difference between CA patients with NWT and increased wall thickness in age (60 vs. 62 years; $\mathrm{p}=0.56)$;) $\mathrm{EF}(60+11$ vs $59+13 ; \mathrm{p}=0.7)$ IgA (72 vs. $56 ; \mathrm{p}=0.07)$; hypertension ( $52 \%$ vs. $33 \%$; $\mathrm{p}=0.8)$, diabetes $(17 \%$ vs. $22 \%$; $\mathrm{p}=0.9)$;chronic kidney disease $(43 \%$ vs $28 \% ; \mathrm{p}=0.2)$ and low voltage EKG ( $23 \%$ vs. $26 \%$; $p=0.7$ ). There was a difference between the two groups in the following variables: female gender $(60 \%$ vs. $23 \%$; p- 0.007$)$, WT $(0.97+0.1$ vs. $1.5+0.2 ; \mathrm{P}<0.0001)$ EDV $(109+55$ vs $86+45 ; \mathrm{p}=0.05)$; BNP ( 249 vs $629 ; \mathrm{p}=0.01)$; lambda light chains (6.8 vs 2.6 ; $\mathrm{p}=0.04)$. There was no difference in the median survival between the two groups (50\%vs. $52 \%$; log rank p value $=0.9$ ); median survival times ( 1215 vs. 1450 days; $\mathrm{p}=0.6$ ).

Cardiology, UAMS, Little Rock, AR, USA zero/1.0/) applies to the data made available in this article, unless otherwise stated. 